Research

Open Access

\title{
Time course of plasma gelsolin concentrations during severe sepsis in critically ill surgical patients
}

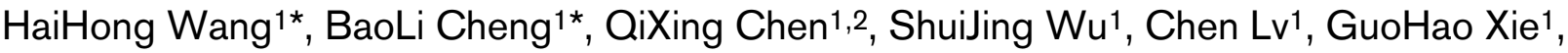 \\ Yue Jin ${ }^{1}$ and XiangMing Fang ${ }^{1}$
}

\author{
1Department of Anesthesiology, the First Affiliated Hospital, School of Medicine, Zhejiang University, QingChun Road, Hangzhou 310003, PR China \\ ${ }^{2}$ Key Laboratory of Multiple Organ Transplantation, Ministry of Public Health, the First Affiliated Hospital, School of Medicine, Zhejiang University, \\ QingChun Road, Hangzhou 310003, PR China \\ ${ }^{*}$ Contributed equally \\ Corresponding author: XiangMing Fang, xiangming_fang@163.com
}

Received: 12 Apr 2008 Revisions requested: 30 May 2008 Revisions received: 7 Aug 2008 Accepted: 17 Aug 2008 Published: 17 Aug 2008

Critical Care 2008, 12:R106 (doi:10.1186/cc6988)

This article is online at: http://ccforum.com/content/12/4/R106

(c) 2008 Wang et al.; licensee BioMed Central Ltd.

This is an open access article distributed under the terms of the Creative Commons Attribution License (http://creativecommons.org/licenses/by/2.0), which permits unrestricted use, distribution, and reproduction in any medium, provided the original work is properly cited.

\begin{abstract}
Introduction Gelsolin is an actin-binding plasma protein that is part of an 'actin-scavenging' system. Studies suggest that plasma gelsolin may play a crucial role in the pathophysiology of sepsis. Little is known about the course of plasma gelsolin levels over time in patients with severe sepsis. The aim of the study was to investigate plasma gelsolin levels in severe septic patients and to determine whether these levels predict the severity or clinical outcome of severe sepsis.

Methods Ninety-one patients who were diagnosed with severe sepsis at admission to a surgical intensive care unit were enrolled, and admission plasma gelsolin levels were recorded. Plasma gelsolin levels were recorded daily in 23 of these patients. Daily plasma gelsolin levels were recorded in an additional 15 nonseptic critically ill patients. Fifteen volunteers served as healthy control individuals. Plasma gelsolin levels were measured using an enzyme-linked immunosorbent assay. Concentrations of IL-6, IL-10 and tumour necrosis factor (TNF)$\alpha$ were also measured on intensive care unit admission.
\end{abstract}

Results The admission gelsolin levels were significantly decreased in severe sepsis $(20.6 \pm 11.7 \mathrm{mg} / \mathrm{l})$ compared with nonseptic critically ill patients $(52.3 \pm 20.3 \mathrm{mg} / \mathrm{l} ; P<0.001)$ and healthy control individuals $(126.8 \pm 32.0 \mathrm{mg} / \mathrm{l} ; P<0.001)$. Severe septic patients had increased IL- 6 levels compared with nonseptic critically ill patients $(20.0 \pm 10.7 \mathrm{pg} / \mathrm{ml}$ versus $11.4 \pm$ $13.9 \mathrm{pg} / \mathrm{ml} ; P=0.048$ ), whereas no significant difference in IL10 or TNF- $\alpha$ levels was observed (IL-10: $97.9 \pm 181.5 \mathrm{pg} / \mathrm{ml}$ versus $47.4 \pm 91.5 \mathrm{pg} / \mathrm{ml}$, respectively $[P=0.425]$; TNF- $\alpha$ : $14.2 \pm 13.9 \mathrm{pg} / \mathrm{ml}$ versus $6.9 \pm 5.3 \mathrm{pg} / \mathrm{ml}$, respectively; $P=$ 0.132 ). Survivors of severe sepsis exhibited substantial recovery of their depressed plasma gelsolin levels, whereas gelsolin levels in nonsurvivors remained at or below their depleted admission levels.

Conclusion Plasma gelsolin may be a valuable marker for severe sepsis. Recovery of depleted plasma gelsolin levels correlated with clinical improvement. The prognostic role of plasma gelsolin in critical illness requires further investigation in a large cohort.

\section{Introduction}

Gelsolin, a protein of 82 to $84 \mathrm{kDa}$, is a member of gelsolin protein superfamily, which exists in a cytoplasmic as well as an excreted plasma isoform, and contains six homologous repeats termed gelsolin-like $(G)$ domains [1-3]. Plasma gelsolin is the principal circulating protein able to sever and scavenge circulating filamentous actin [4-6], which may enhance some major components of proinflammatory cytokine production, impair the microcirculation and compromise multiple organs [7-10]. In animal models, plasma gelsolin appears to be beneficial, possibly by virtue of its ability to counteract the pathophysiological consequences of actin release during trauma, injury and infection [11-14].

In animal models of sepsis, depletion of plasma gelsolin correlates with elevated circulating levels of actin and gelsolin replacement modifies the cytokine profile and improves survival [14]. In humans the plasma gelsolin levels are markedly 
decreased in acute liver failure, myocardial infarction, septic shock, myonecrosis and allogeneic stem cell transplantation, and the degree of depletion correlates with the degree of organ dysfunction, as measured using disease-specific markers $[15,16]$. Soon after traumatic injury, plasma concentrations of gelsolin are significantly reduced compared with those in healthy individuals [17]. Admission plasma gelsolin levels in patients admitted with a variety of critical illness were associated with the development of acute respiratory distress syndrome and septic shock [15,17]. Lee and coworkers [18] serially measured plasma gelsolin levels in patients after surgery or trauma for 5 days and demonstrated that the decreased plasma gelsolin levels seen in such patients are stable over this period. However, that study did not assess the time course of gelsolin recovery or its correlation with clinical improvement.

We studied plasma gelsolin levels at the time of admission to the intensive care unit (ICU) in patients with severe sepsis. Additionally, we measured daily plasma gelsolin levels in critically ill patients admitted to a surgical ICU with severe sepsis and without sepsis. Plasma gelsolin levels were also measured in a cohort of healthy volunteers. Gelsolin levels were compared among these groups and changes in these levels were observed over time to determine whether these changes were associated with outcomes in patients with severe sepsis.

\section{Materials and methods}

This study was performed in accordance with the ethical guidelines of the School of Medicine, Zhejiang University. The protocol was approval by the Institutional Review Board (Ethics Committee). Written informed consent was obtained from both patients and healthy volunteers. In cases in which consent was obtained from the relatives of patients who were unable to give it, consent was later obtained from patients who regained the ability to do so. Patients in this prospective observational study were cared for in a surgical ICU at a university hospital. Patients with severe sepsis were enrolled upon admission to the ICU, using the criteria of the American College of Chest Physician/Society of Critical Care Medicine Consensus Conference Committee [19]. Exclusion criteria were any of the following: lack of informed consent, age younger than 18 years, and pre-existing immunological or haematological diseases. In addition to demographic information, Acute Physiology and Chronic Health Evaluation II (APACHE II) [20] scores and Sequential Organ Failure Assessment (SOFA) [21] were recorded for all patients with severe sepsis. Deaths were defined as all-cause, in-hospital deaths. Fifteen nonseptic critically ill patients at the same ICU during the study period were enrolled randomly as the critically ill control group. In addition, 15 volunteers served as the healthy control group. All patients and volunteers were of Chinese Han origin.

Whole blood samples were obtained from 91 consecutive patients with severe sepsis from 1 June 2006 to 31 May 2007 within 24 hours after ICU admission. Among these patients, daily plasma gelsolin levels were measured in 23 consecutive patients. Day 0 was defined as the time of admission into the surgical ICU. Whole blood samples were also obtained daily from 15 nonseptic, critically ill patients at ICU admission and the following 5 consecutive days. A single whole blood sample was obtained from each of the 15 healthy volunteers. Whole blood samples $(3 \mathrm{ml})$ were collected into EDTA-containing tubes. After being centrifuged at $2,500 \mathrm{~g}$ for 5 minutes, plasma was harvested and frozen at $-80^{\circ} \mathrm{C}$ until analysis. Plasma gelsolin levels were measured using an enzyme-linked immunosorbent assay, in accordance with the manufacturer's instructions (CoTimes, Beijing, China). Admission plasma tumour necrosis factor (TNF)- $\alpha, \mathrm{IL}-6, \mathrm{IL}-10$ and albumin levels were determined using enzyme-linked immunosorbent assay, in accordance with the manufacturer's instructions (R\&D systems, Minneapolis, MN, USA) in both the severe sepsis group and the nonseptic critically ill group.

Normally distributed data are presented as mean \pm standard deviation and compared using Student's $t$-test. Non-normally distributed data are presented as median and interquartile range, and compared using Mann-Whitney U-test. For consistency with previous studies, some variables with unknown/nonnormal distribution (for instance, plasma level of TNF- $\alpha$, IL-6, and so on) were presented as mean \pm standard deviation. Noncontinuous variables are presented as percentages and were analyzed using $\chi^{2}$ test or Fisher's exact test. A logistic regression was performed with the occurrence of severe sepsis as the dependent factor and admission plasma gelsolin, IL6 , IL-10, TNF- $\alpha$, albumin, age and sex as independent factors. The Forward methodology was adopted in the regression process. A variable would enter the model with a $P$ value under 0.05 and would be removed with a $P$ value greater than 0.10 . All statistical analysis was performed with SPSS 14.0 for Windows (SPSS, Chicago, IL, USA). P values under 0.05 (twotailed) were considered statistically significant.

\section{Results}

Of the 91 patients with severe sepsis enrolled in this study, 51 $(56.0 \%)$ patients died in hospital. The underlying diseases associated with the development of severe sepsis were nosocomial pneumonia ( $n=31)$, bowel obstruction $(n=14)$, severe acute pancreatitis $(n=14)$, intestinal or gastric perforation $(n=10)$, trauma $(n=10)$, infection of liver or biliary tree $(n=6)$ and others $(n=6)$. The demographic and clinical data for the patients are presented in Table 1. Among the 15 nonseptic critically ill control patients there were no deaths. No difference was observed in age and sex between nonseptic critically ill patients and patients with severe sepsis.

As shown in Figure 1, the plasma gelsolin level at the time of admission in the severe sepsis group was $20.6 \pm 11.7 \mathrm{mg} / \mathrm{l}$, which was significantly lower than that of 15 nonseptic critically ill patients $(52.3 \pm 20.3 \mathrm{mg} / \mathrm{l}, P<0.001)$. The admission 
Table 1

\begin{tabular}{|c|c|c|c|}
\hline \multirow[t]{2}{*}{ Characteristic } & \multicolumn{2}{|c|}{ Patients with severe sepsis } & \multirow[t]{2}{*}{$P$} \\
\hline & Nonsurviving $(n=51)$ & Surviving $(n=40)$ & \\
\hline Age (years) & 68 (48 to 75$)$ & 57.5 (44.3 to 72.8$)$ & 0.15 \\
\hline Males (n [\%]) & $29(56.9)$ & $27(67.5)$ & 0.386 \\
\hline Plasma concentration of gelsolin (mg/l; mean \pm SE) & $20.9 \pm 11.2$ & $20.2 \pm 12.3$ & 0.786 \\
\hline Plasma concentration of IL-6 (pg/ml; mean \pm SE) & $21.6 \pm 11.6$ & $19.0 \pm 10.0$ & 0.59 \\
\hline Plasma concentration of IL-10 (pg/ml; mean \pm SE) & $68.7 \pm 56.0$ & $127.3 \pm 250.8$ & 0.347 \\
\hline Plasma concentration of TNF- $\alpha(\mathrm{pg} / \mathrm{ml}$; mean \pm SE) & $19.2 \pm 17.7$ & $9.2 \pm 5.4$ & 0.033 \\
\hline Plasma concentration of albumin ( $g / l ;$ mean $\pm \mathrm{SE}$ ) & $27.5 \pm 6.2$ & $25.1 \pm 6.5$ & 0.101 \\
\hline APACHE II (median [IQR]) & 24 (17.8 to 27.3$)$ & 17 (12.5 to 22$)$ & $<0.001$ \\
\hline Severe SOFA (median [IQR]) & 9 (6 to 13.3$)$ & $6(4$ to 8$)$ & 0.002 \\
\hline \multicolumn{4}{|l|}{ Organs with acute dysfunction ( $[\%]$ ) } \\
\hline Respiratory & $48(94.1 \%)$ & $32(80 \%)$ & 0.054 \\
\hline Cardiovascular & $20(39.2 \%)$ & $6(\%)$ & 0.018 \\
\hline Renal & $18(35.3 \%)$ & $6(15 \%)$ & 0.033 \\
\hline Haematologic & $20(39.2 \%)$ & $14(35 \%)$ & 0.827 \\
\hline Central nervous system & $35(68.6 \%)$ & $23(57.5 \%)$ & 0.283 \\
\hline Hepatic & $24(47.1 \%)$ & $14(35 \%)$ & 0.288 \\
\hline
\end{tabular}

APACHE, Acute Physiology and Chronic Health Evaluation II; IQR, interquartile range; SE, standard error; SOFA, Sequential Organ Failure Assessment; TNF, tumour necrosis factor.

levels of plasma gelsolin in both severe sepsis and nonseptic critically ill patients were significantly different from those of the 15 healthy control individuals $(126.8 \pm 32.0 \mathrm{mg} / \mathrm{l} ; P<$ $0.001)$. Severe septic patients exhibited an increased IL-6

\section{Figure 1}

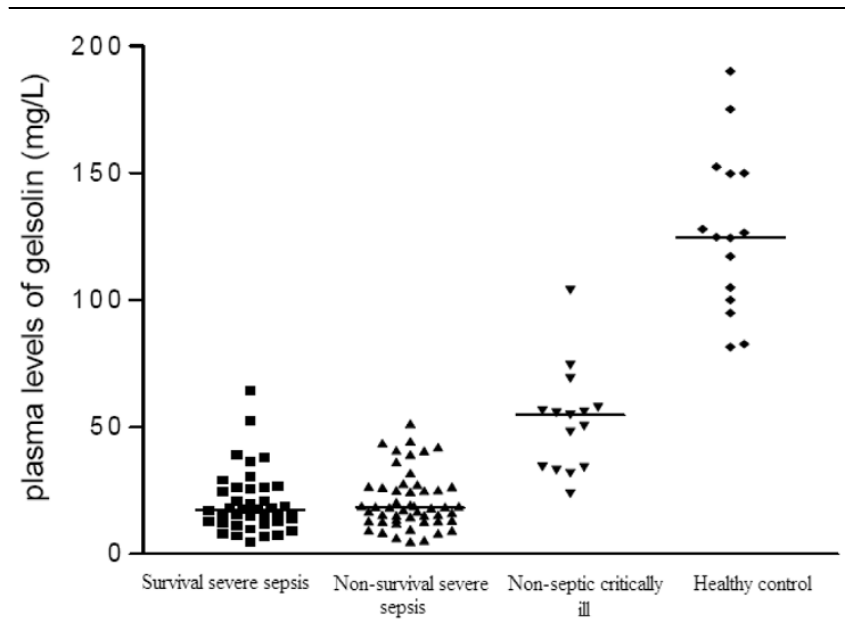

Admission plasma gelsolin levels. Presented is a comparision of the plasma gelsolin levels at the time of admission of survival severe sepsis, nonsurvival severe sepsis, nonseptic critically ill and healthy control. level compared with the nonseptic critically ill patients $(20.0 \pm$ $10.7 \mathrm{pg} / \mathrm{ml}$ versus $11.4 \pm 13.9 \mathrm{pg} / \mathrm{ml} ; P=0.048$ ), whereas no significant differences in IL-10 and TNF- $\alpha$ levels were observed between the two groups (IL-10: $97.9 \pm 181.5 \mathrm{pg} / \mathrm{ml}$ versus $47.4 \pm 91.5 \mathrm{pg} / \mathrm{ml}$, respectively $[P=0.425]$; TNF- $\alpha$ $14.2 \pm 13.9 \mathrm{pg} / \mathrm{ml}$ versus $6.9 \pm 5.3 \mathrm{pg} / \mathrm{ml}$, respectively $[P=$ $0.132])$. Both the severe sepsis group and the nonseptic critically ill group had similar lowered plasma albumin levels (26.4 $\pm 6.4 \mathrm{~g} / \mathrm{l}$ versus $29.2 \pm 3.9 \mathrm{~g} / \mathrm{l} ; P=0.071)$. Higher $(\geq 25)$ APACHE II scores were associated with lower plasma gelsolin levels at ICU admission, as compared with lower $(<25)$ scores (17.1 $\pm 9.1 \mathrm{mg} / \mathrm{l}$ versus $22.4 \pm 14.4 \mathrm{mg} / \mathrm{l} ; P=0.044)$. In contrast, higher $(\geq 8)$ admission SOFA scores were not associated with plasma gelsolin levels as compared with patients with lower $(<8)$ score $(21.5 \pm 12.8 \mathrm{mg} / \mathrm{l}$ versus $19.7 \pm 10.6$ $\mathrm{mg} / \mathrm{l} ; P=0.457)$.

Logistic regression revealed that among the seven candidate risk factors (admission plasma gelsolin, IL-6, IL-10, TNF- $\alpha$, albumin, age and sex), admission plasma gelsolin was the only independent factor able to predict the occurrence of severe sepsis. However, there was no significant difference in the gelsolin levels between surviving and nonsurviving patients with severe sepsis $(20.2 \pm 12.3 \mathrm{mg} / \mathrm{l}$ versus $20.9 \pm 11.2 \mathrm{mg} / \mathrm{l} ; P=$ 0.786). 
Twenty-three patients with severe sepsis and 15 nonseptic critically ill patients had daily plasma gelsolin levels measured consecutively after their ICU admission. Nine out of 23 consecutive septic patients who had been sampled for gelsolin levels daily stayed in the ICU for longer than 14 days. The time course of plasma gelsolin concentration in these nine septic patients is shown in Figure 2. Among survivors depressed plasma gelsolin levels appeared to recover after day 11, whereas plasma gelsolin levels remained low or even decreased further in the nonsurvivors with severe sepsis. For nonseptic critically ill patients, the depressed plasma gelsolin levels increased after day 3 of the surgical ICU stay, which was coincided with clinical improvement.

\section{Discussion}

In the present study we found that admission plasma gelsolin levels were lower in patients with severe sepsis than in nonseptic critically ill ICU patients and healthy control individuals. Admission plasma gelsolin level was a independent risk factor that correlated with occurrence of severe sepsis, although it did not significantly differ between surviving and nonsurviving patients with severe sepsis. Recovery of plasma gelsolin levels was observed late in the course in survivors but not in nonsurvivors with severe sepsis.

In the study conducted by Lee and coworkers [14], depletion of plasma gelsolin in animal models of sepsis occurred 6 hours after a septic challenge with either endotoxin (lipopolysaccharide) or a polymicrobial challenge after caecal-ligation and puncture [14]. Several clinical studies $[15,16]$ have observed

Figure 2

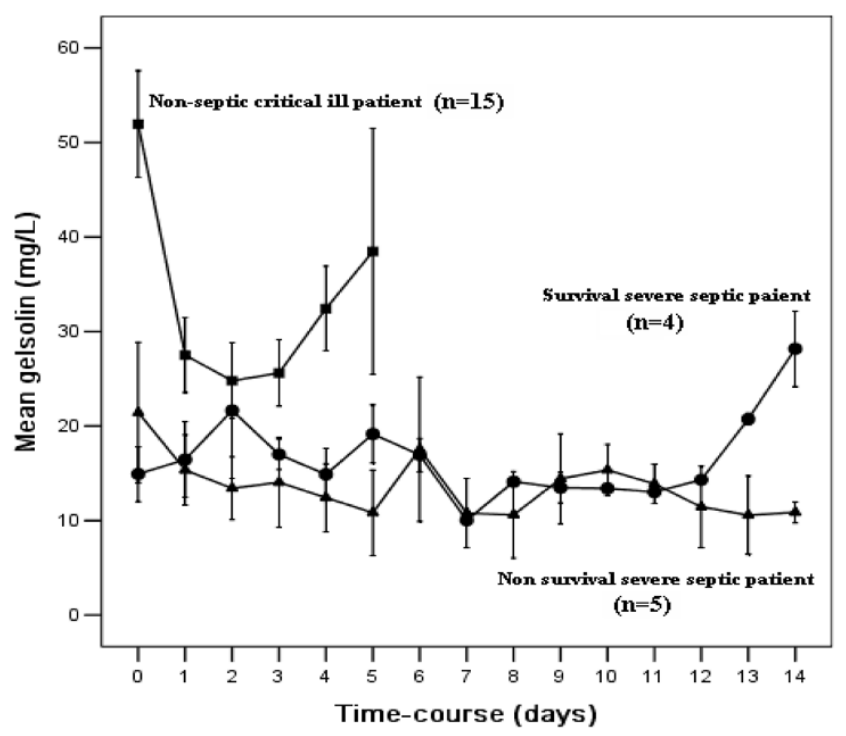

Time course of plasma gelsolin levels. Presented are the courses over time of plasma gelsolin levels in nonseptic critically ill patients, and patients with severe sepsis who survived and did not survive. Results are expressed as means \pm standard error. that a low gelsolin level after an initial insult such as injury or inflammation reflected greater severity of disease and poorer outcomes. The depletion of plasma gelsolin soon after a septic challenge may result from exposure of the actin cytoskeleton, which occurs as part of cellular injury [22-24]. In turn, depletion of gelsolin could allow the formation of actin filaments, which would lead to further tissue injury and organ dysfunction [7-10,25]. In addition, plasma gelsolin binds bioactive inflammatory mediators including lipopolysaccharide [26], lysophosphatidic acid [27] and platelet-activating factor [28].

The present study revealed that plasma gelsolin levels measured at the time of ICU admission in patients with severe sepsis were lower than those in nonseptic critically ill patients and healthy control individuals. Although plasma albumin levels in both severe septic group and nonseptic critically ill group were below the normal value, there was no significant difference between the two groups. This indicates that the decrease in plasma gelsolin level was specific and not a simple consequence of systemic plasma protein loss or dilution. Combined with previous reports [14-18], this study suggests that early determination of plasma gelsolin level could facilitate early diagnosis of severe sepsis.

In contrast to the study conducted by Mounzer and coworkers [17], this study could not replicate a definite association between plasma gelsolin levels of admission and mortality. Mounzer and coworkers demonstrated that low plasma gelsolin levels at admission were associated with increased risk for adverse outcomes, including prolonged length of hospital stay and death, in patients who had undergone surgery or who had suffered trauma [17]. Possibly, the characteristics of patient population and the limited number of cases contributed to the conflicted results. Interestingly, Huang and colleagues [10] found that the plasma gelsolin level recovered at the time of clinical improvement. In the present study, among the 15 nonseptic critically ill patients that admitted to the surgical ICU for postoperative or post-traumatic observation, the decreased plasma gelsolin levels demonstrated a recovery after day 3 . Furthermore, this study demonstrated that the depletion of plasma gelsolin recovered with clinical improvements in survivors of severe sepsis, whereas the gelsolin level in nonsurvivors remained low. This finding is consistent with the hypothesis proposed by Lee and coworkers [14], namely that plasma gelsolin can modify systemic inflammatory response and improve the outcome of sepsis via its binding and neutralizing inflammatory mediators during the course of sepsis. To our knowledge, this is the first study to examine the time course of plasma gelsolin changes and its correlation with clinical improvement in septic patients.

The limitations of the study were as follows. The number of patients with severe sepsis enrolled in the time course study is inadequate to allow definitive conclusions to be drawn, and the study does not unequivocally elucidate the role played by 
plasma gelsolin in sepsis or the association of plasma gelsolin with cytokines. Studies such as this one are important because the animal data suggest that repletion of low plasma gelsolin levels may be a useful adjuvant therapy, and it is critical that we detemine means to identify those patients who could potentially benefit from such therapy, if we are to optimize recombinant drug treatment in this setting. Further study is required to address these issues.

\section{Conclusion}

This study suggests that plasma gelsolin levels are a valuable marker of severe sepsis in surgical ICUs. Admission plasma gelsolin levels correlated with severity of sepsis, whereas recovery of plasma gelsolin levels correlated with clinical improvement. The prognostic role played by plasma gelsolin level in critical illness needs to be further investigated in a large cohort.

\section{Key messages}

- Admission plasma gelsolin levels in patients with severe sepsis were lower than those in nonseptic, critically ill ICU patients or healthy control individuals.

- Admission plasma gelsolin levels were associated with the occurrence of severe sepsis.

- Survivors of severe sepsis exhibited substantial recovery of their depressed plasma gelsolin levels, whereas the gelsolin levels in nonsurvivors remained at or below their depleted admission levels.

- Plasma gelsolin may be a valuable marker for severe sepsis.

\section{Competing interests}

The authors declare that they have no competing interests.

\section{Authors' contributions}

$\mathrm{HHW}$ and BLC contributed equally to the manuscript. HHW, BLC, QXC and XMF contributed to the design of the study and drafted the manuscript. HHW, BLC, QHX, SJW and YJ enrolled the patients and participated in the laboratory work. $\mathrm{HHW}, \mathrm{BLC}$ and CL contributed to data analysis and interpretation of the results. All authors read and approved the final manuscript.

\section{Acknowledgements}

This work was financially supported by Program for New Century Excellent Talents in University (XMF; No. NCET-05-0522) and by Zhejiang Provincial Program for the Cultivation of High-level Innovative Health talents (XMF). The authors acknowledge Richard Straube, MD and MSc, for his help in manuscript revision.

\section{References}

1. Yin HL, Stossel TP: Control of cytoplasmic actin gel-sol transformation by gelsolin, a calcium-dependent regulatory protein. Nature 1979, 281:583-586.
2. Kwiatkowski DJ, Stossel TP, Orkin SH, Mole JE, Colten HR, Yin $\mathrm{HL}$ : Plasma and cytoplasmic gelsolins are encoded by a single gene and contain a duplicated actin-binding domain. Nature 1986, 323:455-458.

3. Sun $\mathrm{HQ}$, Yamamoto M, Mejillano M, Yin HL: Gelsolin, a multifunctional actin regulatory protein. J Biol Chem 1999, 274:33179-33182.

4. Janmey PA, Lind SE: Capacity of human serum to depolymerize actin filaments. Blood 1987, 70:524-530.

5. Kwiatkowski DJ, Mehl R, Izumo S, Nadal-Ginard B, Yin HL: Muscle is the major source of plasma gelsolin. J Biol Chem 1988, 263:8239-8243.

6. Lind SE, Smith DB, Janmey PA, Stossel TP: Role of plasma gelsolin and the vitamin D-binding protein in clearing actin from the circulation. J Clin Invest 1986, 78:736-742.

7. Haddad JG, Harper KD, Guoth M, Pietra GG, Sanger JW: Angiopathic consequences of saturating the plasma scavenger system for actin. Proc Natl Acad Sci USA 1990, 87:1381-1385.

8. Lind SE, Smith DB, Janmey PA, Stossel TP: Depression of gelsolin levels and detection of gelsolin-actin complexes in plasma of patients with acute lung injury. Am Rev Respir Dis 1988, 138:429-434.

9. Rosengart MR, Arbabi S, Bauer GJ, Garcia I, Jelacic S, Maier RV: The actin cytoskeleton: an essential component for enhanced TNFalpha production by adherent monocytes. Shock 2002, 17:109-113.

10. Huang S, Rhoads SL, DiNubile MJ: Temporal association between serum gelsolin levels and clinical events in a patient with severe falciparum malaria. Clin Infect Dis 1997, 24:951-954

11. Rothenbach PA, Dahl B, Schwartz JJ, O'Keefe GE, Yamamoto M, Lee WM, Horton JW, Yin HL, Turnage RH: Recombinant plasma gelsolin infusion attenuates burn-induced pulmonary microvascular dysfunction. J Appl Physiol 2004, 96:25-31.

12. Christofidou-Solomidou M, Scherpereel A, Solomides CC, Muzykantov VR, Machtay M, Albelda SM, DiNubile MJ: Changes in plasma gelsolin concentration during acute oxidant lung injury in mice. Lung 2002, 180:91-104.

13. Becker PM, Kazi AA, Wadgaonkar R, Pearse DB, Kwiatkowski D, Garcia JG: Pulmonary vascular permeability and ischemic injury in gelsolin-deficient mice. Am J Respir Cell Mol Biol 2003, 28:478-484.

14. Lee PS, Waxman AB, Cotich KL, Chung SW, Perrella MA, Stossel TP: Plasma gelsolin is a marker and therapeutic agent in animal sepsis. Crit Care Med 2007, 35:849-855.

15. Suhler E, Lin W, Yin HL, Lee WM: Decreased plasma gelsolin concentrations in acute liver failure, myocardial infarction, septic shock, and myonecrosis. Crit Care Med 1997, 25:594-598.

16. DiNubile MJ, Stossel TP, Ljunghusen OC, Ferrara JL, Antin JH: Prognostic implications of declining plasma gelsolin levels after allogeneic stem cell transplantation. Blood 2002, 100:4367-4371.

17. Mounzer KC, Moncure M, Smith YR, Dinubile MJ: Relationship of admission plasma gelsolin levels to clinical outcomes in patients after major trauma. Am J Respir Crit Care Med 1999, 160:1673-1681.

18. Lee PS, Drager LR, Stossel TP, Moore FD, Rogers SO: Relationship of plasma gelsolin levels to outcomes in critically ill surgical patients. Ann Surg 2006, 243:399-403.

19. Bone RC, Balk RA, Cerra FB, Dellinger RP, Fein AM, Knaus WA Schein RM, Sibbald WJ: Definitions for sepsis and organ failure and guidelines for the use of innovative therapies in sepsis: The ACCP/SCCM Consensus Conference Committee. American College of Chest Physicians/Society of Critical Care Medicine. Chest 1992, 101:1644-1655.

20. Rowan KM, Kerr JH, Major E, McPherson K, Short A, Vessey MP: Intensive Care Society's APACHE II study in Britain and Ireland - I: variations in case mix of adult admissions to general intensive care units and impact on outcome. BMJ 1993, 307:972-977.

21. Ferreira FL, Bota DP, Bross A, Mélot C, Vincent JL: Serial evaluation of the SOFA score to predict outcome in critically ill patients. JAMA 2001, 286:1754-1758.

22. De Scheerder I, Vandekerckhove J, Robbrecht J, Algoed L, De Buyzere M, De Langhe J, De Schrijver G, Clement D: Postcardiac injury syndrome and an increased humoral immune response 
against the major contractile proteins (actin and myosin). Am J Cardiol 1985, 56:631-633.

23. Goldschmidt-Clermont PJ, Lee WM, Galbraith RM: Proportion of Gc (vitamin D-binding protein] in complexed form: relation to clinical outcome in fulminant hepatic necrosis. Gastroentero/ogy 1988, 94:1454-1458.

24. Smith DB, Janmey PA, Lind SE: Circulating actin-gelsolin complexes following oleic acid-induced lung injury. Am J Pathol 1988, 130:261-267.

25. Lee WM, Galbraith RM: The extracellular actin-scavenger system and actin toxicity. N Engl J Med 1992, 326:1335-1341.

26. Bucki R, Georges PC, Espinassous Q, Funaki M, Pastore JJ, Chaby R, Janmey PA: Inactivation of endotoxin by human plasma gelsolin. Biochemistry 2005, 44:9590-9597.

27. Goetzl EJ, Lee H, Azuma T, Stossel TP, Turck CW, Karliner JS: Gelsolin binding and cellular presentation of lysophosphatidic acid. J Bio Chem 2000, 275:14573-14578.

28. Osborn TM, Dahlgren C, Hartwig JH, Stossel TP: Modifications of cellular responses to lysophosphatidic acid and platelet-activating factor by plasma gelsolin. Am J Physiol Cell Physiol 2007, 292:C1323-C1330. 